This is an author-created, un-copyedited version of an article accepted for publication in 2D Materials. The publisher is not responsible for any errors or omissions in this version of the manuscript or any version derived from it. The Version of Record is available online at https://doi.org/10.1088/2053-1583/1/3/035003.

Polarization doping of graphene on silicon carbide

Samir Mammadov, Jürgen Ristein, Roland J Koch, Markus Ostler, Christian Raidel, Martina Wanke, Remigijus Vasiliauskas, Rositza Yakimova and Thomas Seyller

2D Materials 1 (2014) 035003 


\title{
Polarization Doping of Graphene on Silicon Carbide
}

\author{
Samir Mammadov ${ }^{1}$, Jürgen Ristein ${ }^{2}$, Roland J. Koch ${ }^{1}$, \\ Markus Ostler ${ }^{1}$, Christian Raidel ${ }^{1}$, Martina Wanke ${ }^{1}$, \\ Remigijus Vasiliauskas ${ }^{3}$, Rositza Yakimova ${ }^{3}$, and Thomas \\ Seyller ${ }^{1}$ \\ 1 Institut für Physik, Technische Universität Chemnitz, Reichenhainer Str. 70, \\ 09126 Chemnitz, Germany \\ 2 Lehrstuhl für Laserphysik, Universität Erlangen-Nürnberg, \\ Erwin-Rommel-Str. 1, 91058 Erlangen, Germany \\ 3 Department of Physics, Chemistry and Biology, Linköping University, \\ SE-58183, Linköping, Sweden \\ E-mail: samir.mammadov@physik.tu-chemnitz.de, \\ thomas.seyller@physik.tu-chemnitz.de
}

\begin{abstract}
The doping of quasi-freestanding graphene (QFG) on H-terminated, Si-face $6 \mathrm{H}_{-}, 4 \mathrm{H}-$, and $3 \mathrm{C}-\mathrm{SiC}$ is studied by angle-resolved photoelectron spectroscopy (ARPES) close to the Dirac point. Using semi-insulating as well as n-type doped substrates we shed light on the contributions to the charge carrier density in QFG caused by i) the spontaneous polarization of the substrate, and ii) the band alignment between the substrate and the graphene layer. In this way we provide quantitative support for the previously suggested model of polarization doping of graphene on SiC [Phys. Rev. Lett. 108, 246104 (2012)].
\end{abstract}

Keywords: graphene, silicon carbide, doping, ARPES, XPS

Submitted to: 2D Mater.

\section{Introduction}

Silicon carbide $(\mathrm{SiC})$ crystals have evolved as the substrates of choice for graphene growth [1-3]. Epitaxial graphene (EG) on $\mathrm{SiC}$ has been a subject of intensive research due to various promising applications, such as, e.g., high frequency transistors [4-6], frequency mixers [7] or resistance standards [8]. The process of precisely controlling the number of graphene layers [2] and the determination of the basic parameters of the band structure and its dependence on the number of layers [10] or on interface modification is of great importance for the future of carbon-based nanoelectronics. Although different polytypes such as $3 \mathrm{C}-, 4 \mathrm{H}-$ and $6 \mathrm{H}-\mathrm{SiC}$ are available, such fundamental questions as how the charge carrier concentration and type in the graphene layers varies with the substrate polytype and substrate doping remain largely unanswered. This issue plays a very important role in the research of graphene on $\mathrm{SiC}$, as well as in its industrial applications. In this study we experimentally 
address this issue and create a rational basis for the choice of substrate for future graphene-on-SiC applications.

It is well known that $\mathrm{EG}$ on $\mathrm{SiC}(0001)$, which is situated on top of the socalled buffer layer (denoted by $6 \sqrt{3}$ in the figures of this work) is intrinsically n-type doped $[1,9,10]$. Note that the buffer layer is already a honeycomb lattice of carbon atoms, but on account of hybridization with $\mathrm{SiC}$ states it does not exhibit the Diraclike dispersion at the K-point of the Brillouin zone, [9,11-13]. A model by Kopylov et al. [14] explained the n-type doping by either bulk or interface donor states.

The situation changes when going from EG on the buffer layer to quasifreestanding graphene $(\mathrm{QFG})$ on $\mathrm{H}$-terminated $\mathrm{SiC}(0001)$ [15-17, 19, 19]. The latter is obtained - as was first shown by Riedl et al. $[15,16]$ - by annealing the buffer layer in an atmosphere of hydrogen. Hydrogen intercalates between the SiC surface and the buffer layer, saturating the dangling bonds of the topmost Si atoms [21,22]. In that way, the buffer layer is decoupled from the $\mathrm{SiC}$ surface. On account of the dramatically reduced interaction of the now $\mathrm{H}$-terminated $\mathrm{SiC}(0001)$ surface with the carbon atoms, an almost undisturbed graphene layer emerges which is referred to as quasi-freestanding graphene. For details about the preparation see also section II. In contrast to EG, QFG has an excess of holes. This p-type doping cannot be explained by the model of Kopylov [14]. Instead, it has been attributed to the influence of the spontaneous polarization of the hexagonal $\mathrm{SiC}$ substrate [23].

The spontaneous polarization (which is a bulk property) leads to a polarization charge on the polar surfaces of hexagonal semiconductor compounds, such as $\mathrm{SiC}$ and GaN, which is usually compensated by internal charge formation due to depletion/accumulation of charge carriers or by external buildup of ionic charge on the surfaces. Only the modulation of the polarization charge at a surface upon an external perturbation can be measured [24-26], making the effect itself difficult to address experimentally. The existence of various $\mathrm{SiC}$ polytypes offers an excellent opportunity to address the question. While spontaneous polarization is absent for the cubic polytype 3C-SiC due to symmetry, it is mainly induced at the inversion of the stacking sequence of the $\mathrm{SiC}$ double layers in the hexagonal polytypes. Consequently, varying the hexagonality of the $\mathrm{SiC}$ polytype changes the polarization proportionally. Thus, for $4 \mathrm{H}-\mathrm{SiC}(0001)$ it is expected to be $6 / 4$ times larger than for $6 \mathrm{H}-\mathrm{SiC}(0001)$ [27-30].

In order to test this hypothesis, we have carried out systematic angle-resolved photoelectron spectroscopy (ARPES) measurements close to the Dirac point. In particular, the comparison of the total charge densities of quasi-freestanding graphene (QFG) on $6 \mathrm{H}-\mathrm{SiC}(0001)$, $4 \mathrm{H}-\mathrm{SiC}(0001)$ and $3 \mathrm{C}-\mathrm{SiC}(111)$ provides a quantitative confirmation of the polarization doping proposed earlier [23]. In addition, by comparing n-type and semi-insulating substrates with identical graphene layers on top we provide further additional insights into the question how the charge carrier density in graphene overlayers is influenced by the doping level of the $\mathrm{SiC}$ bulk.

\section{Sample Preparation and Characterization}

EG was formed by sublimation growth in argon atmosphere [2] on the Si-face of various $\mathrm{SiC}$ substrates. Nitrogen doped n-type $6 \mathrm{H}-\mathrm{SiC}(0001)$ was purchased from SiCrystal AG while semi-insulating $6 \mathrm{H}-\mathrm{SiC}$ wafers were obtained from II-VI Inc. Semi-insulating as well as N-doped, n-type $4 \mathrm{H}-\mathrm{SiC}$ wafers were purchased from and Cree Inc. Finally, a sample of $3 \mathrm{C}-\mathrm{SiC}$ with (111) surface orientation grown on a $6 \mathrm{H}-\mathrm{SiC}(0001)$ substrate was prepared at Linköping University. Growth of EG (including samples covered by 
Polarization Doping of Graphene on Silicon Carbide

Table 1. Parameters (annealing temperature $T$, Argon pressure $p_{A r}$, Ar flow rate $\phi_{A r}$, hydrogen pressure $p_{H_{2}}$, hydrogen flow rate $\phi_{H_{2}}$, and annealing time) used for the preparation of the samples. Note that parameters vary between different growth setups so that the values given here cannot be transferred directly to other equipment.

\begin{tabular}{lllll}
\hline \multicolumn{5}{c}{ Growth of EG } \\
\hline Sample & $\mathrm{T}\left({ }^{\circ} \mathrm{C}\right)$ & $p_{A r} / \mathrm{mbar}$ & $\phi_{A r} / \mathrm{slm}$ & $t / \mathrm{min}$ \\
\hline $6 \sqrt{3}$ & 1450 & 1000 & 1.0 & 15 \\
MLG & 1650 & 1000 & 1.0 & 15 \\
BLG & 1750 & 400 & 1.0 & 15 \\
TLG & 1800 & 400 & 1.0 & 15 \\
\hline \multicolumn{5}{c}{ Intercalation } \\
\hline Sample & $\mathrm{T}\left({ }^{\circ} \mathrm{C}\right)$ & $p_{H_{2}} / \mathrm{mbar}$ & $\phi_{H_{2}} / \mathrm{slm}$ & $t / \mathrm{min}$ \\
\hline QFMLG & 540 & 900 & 0.9 & 90 \\
QFBLG & 840 & 900 & 0.9 & 90 \\
QFTLG & 880 & 900 & 0.9 & 120 \\
\hline
\end{tabular}

* substrate with off axis angle $\geq 0.3^{\circ}$

the buffer layer only) was performed using a custom built reactor which is described elsewhere [31]. By adjusting the temperature and the Ar pressure, the thickness of EG can be controlled from the pure buffer layer $(6 \sqrt{3})$ to up to three monolayers on the buffer layer. The different EG samples are denoted as MLG, BLG, and TLG for one, two, or three layers of graphene on top of the buffer layer, respectively. Growth parameters are summarized in table 1.

QFG was prepared by annealing in ultra-pure hydrogen [15-18] using a custom build setup and parameters also listed in table 1. As mentioned above, hydrogen saturates the $\mathrm{SiC}$ surface, thereby releasing the buffer layer. Hence, carrying out $\mathrm{H}$ intercalation with a sample that consists only of the buffer layer, quasi-freestanding monolayer graphene (QFMLG) is obtained. In the same way, MLG and BLG are converted into quasi-freestanding bilayer graphene (QFBLG) and quasi-freestanding trilayer graphene (QFTLG), respectively. In other words, the conversion of the buffer layer adds one graphene layer to the stack. Note that this process is not hydrogenation of graphene, as only the $\mathrm{SiC}$ substrate surface is saturated with hydrogen.

Following preparation the samples were characterized with photoelectron spectroscopy. X-ray induced photoelectron spectroscopy (XPS) was carried out using a SPECS PHOIBOS 150 MCD 9 analyzer in conjunction with a monochromatized Al $K_{\alpha}$ source (Specs FOCUS 500). ARPES spectra were collected with the help of a SPECS PHOIBOS 100 2D CCD system operated at beam line UE56/2-PGM1 of the synchrotron radiation source BESSY II. All measurements were carried out at a pressure of $1 \times 10^{-10}$ mbar or lower. While XPS was performed at room temperature, the ARPES data was collected with the sample held at a temperature of $80 \mathrm{~K}$. Prior to the measurements, the ex-situ prepared samples were carefully annealed at $350{ }^{\circ} \mathrm{C}$ to remove possible contaminations from exposure to air. Note that a comparative study like the present one can only be carried out when identical preparation techniques and analytical tools are used. 


\section{Results and Discussion}

\subsection{XPS Analysis}

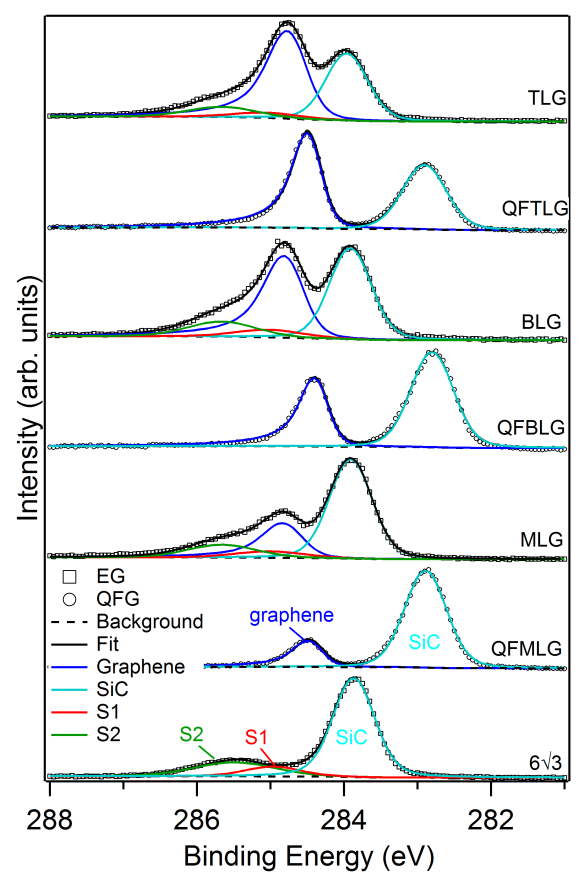

Figure 1. (Color online) $\mathrm{C} 1 \mathrm{~s}$ core-level spectra of graphene on $6 \mathrm{H}-\mathrm{SiC}(0001)$ before $(6 \sqrt{3}$, MLG, BLG) and after H-intercalation (QFMLG, QFBLG, QFTLG). Each spectrum is analyzed in terms of components arising from the $\mathrm{SiC}$ substrate ('SiC'), the graphene ('graphene'), and (where applicable) from the two buffer layer components ('S1', 'S2'). In addition, the spectrum of trilayer graphene (TLG) is also shown for comparison.

The process of conversion of EG films to QFG layers is illustrated in Figure 1 by a series of XPS spectra. The C1s core-level spectra shown in that figure are comprised of various components, which can be attributed to $\mathrm{C}$ atoms in the $\mathrm{SiC}$ substrate ('SiC'), the buffer layer ('S1' and 'S2' [9]), and the graphene layer ('graphene'). It is clear from Figure 1 that in all three cases components S1 and S2 vanish after H-intercalation, which confirms the transformation from epitaxial to quasi-freestanding graphene. The shift of the $\mathrm{SiC}$ signal upon $\mathrm{H}$-intercalation is caused by a different surface band bending which will be commented on later. Most importantly, the position of the graphene component also differs depending on the interface. For EG on the buffer layer, the position is at a higher binding energy than for QFG. In fact, the C1s core line of graphene indicates already a change from n-type doping for EG to p-type doping for QFG, as already observed before [15-18]. The experimental uncertainty of the XPS core level spectra (approximately $\pm 0.1 \mathrm{eV}$ ) is, however, too large to perform a more precise analysis of the charge carrier concentration. This will be done with the help of the ARPES measurements described in the next section. 


\section{2. $A R P E S$}

Figure 2 shows ARPES measurements of QFG on n-type (bulk carrier concentration of $n \approx 5 \times 10^{17} \mathrm{~cm}^{-3}$ ) and semi-insulating (bulk carrier concentration of less than $\left.10^{14} \mathrm{~cm}^{-3}\right) 6 \mathrm{H}-\mathrm{SiC}(0001)(\mathrm{a})-(\mathrm{d})$ and $4 \mathrm{H}-\mathrm{SiC}(0001)(\mathrm{e})-(\mathrm{h})$ substrates. As expected, the number of $\pi$-bands increases from one for QFMLG to two for QFBLG. It is obvious that the Dirac point $\left(E_{\mathrm{D}}\right)$ is located above the Fermi energy $\left(E_{\mathrm{F}}\right)$ for all hexagonal substrates. In order to learn more about the position of $E_{\mathrm{D}}$, the observed bands were fitted with a tight binding model as already used in earlier work [10]. The Fermi velocity turns out to be $v_{\mathrm{F}}=(0.98-1.0) \times 10^{8} \mathrm{~cm} / \mathrm{s}$ and the interlayer hopping matrix element, which is important for bilayers, is $\gamma=(0.38-0.40) \mathrm{eV}$, in agreement with previous work [10]. The position of the bands observed for QFBLG indicates the presence of a small band gap due to an asymmetry $\Delta E=\left|E_{2}-E_{1}\right|$ in the onsite Coulomb potentials $E_{1 / 2}$ on the two layers [32,33]. The band gap is above $E_{\mathrm{F}}$ and therefore not directly visible in ARPES. However, recent two-photon photo emission (2PPE) experiments [34] has given direct evidence for its existence. The fitting parameters are collected in Table 2.
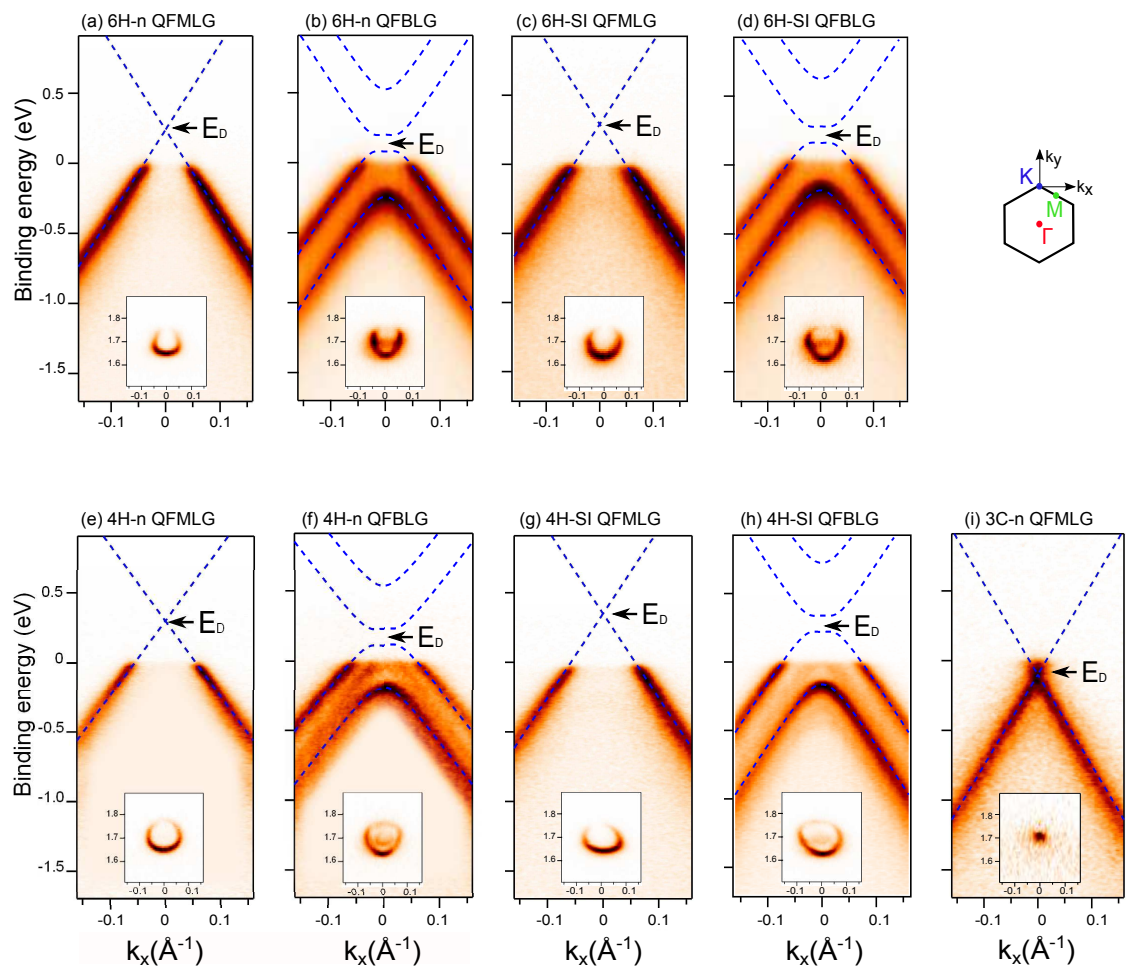

Figure 2. (Color online) $\pi$-bands near $E_{\mathrm{F}}$ for QFMLG and QFBLG on (a,b) n-type $6 \mathrm{H}-\mathrm{SiC}(0001)$, (c,d) semi-insulating $6 \mathrm{H}-\mathrm{SiC}(0001)$ and (e,f) n-type $4 \mathrm{H}-$ $\mathrm{SiC}(0001)$ and $(\mathrm{g}, \mathrm{h})$ semi-insulating $4 \mathrm{H}-\mathrm{SiC}(0001)$ and (i)n-type 3C-SiC(111). The photon energy was $\hbar \omega=95 \mathrm{eV}$. The blue lines show fitted TB bands. The insets depict the corresponding Fermi surfaces. Schematic representation of graphene in reciprocal space on the right shows directions of $k_{x}$ and $k_{y}$ vectors.

The hole density $p$ (per unit cell) was determined from the $\pi$-band Fermi surface 
Polarization Doping of Graphene on Silicon Carbide

Table 2. Properties of $\mathrm{QFG}$ on the different $\mathrm{SiC}$ substrates. $E_{\mathrm{D}}$ is the position of the Dirac point with respect to $E_{\mathrm{F}}$ determined by fitting a tight-binding model to the ARPES data. $\Delta E$ is the difference in the onsite Coulomb potentials and $\gamma$ the interlayer coupling constant of the QFBLG samples. $p$ is the hole concentration. Its value is given per unit cell and per $\mathrm{cm}^{2}$.

\begin{tabular}{llllll}
\hline Sample & $E_{\mathrm{D}} / \mathrm{eV}$ & $\Delta E / \mathrm{eV}$ & $\gamma / \mathrm{eV}$ & $p /$ unit cell & $p / \mathrm{cm}^{2}$ \\
\hline QFMLG/6H-n & 0.24 & & & $2.2 \times 10^{-3}$ & $4.2 \times 10^{12}$ \\
QFBLG/6H-n & 0.14 & 0.12 & 0.38 & $2.3 \times 10^{-3}$ & $4.4 \times 10^{12}$ \\
QFMLG/6H-SI & 0.28 & & & $3.3 \times 10^{-3}$ & $6.2 \times 10^{12}$ \\
QFBLG/6H-SI & 0.22 & 0.12 & 0.38 & $3.5 \times 10^{-3}$ & $6.5 \times 10^{12}$ \\
QFMLG/4H-n & 0.30 & & & $3.6 \times 10^{-3}$ & $6.9 \times 10^{12}$ \\
QFBLG/4H-n & 0.22 & 0.12 & 0.38 & $3.8 \times 10^{-3}$ & $7.2 \times 10^{12}$ \\
QFMLG/4H-SI & 0.34 & & & $4.5 \times 10^{-3}$ & $8.6 \times 10^{12}$ \\
QFBLG/4H-SI & 0.28 & 0.12 & 0.40 & $4.6 \times 10^{-3}$ & $8.7 \times 10^{12}$ \\
QFMLG/3C-n & -0.1 & & & $-0.4 \times 10^{-3}$ & $-0.7 \times 10^{12}$ \\
\hline
\end{tabular}

area $A_{\mathrm{F}}$ from figure 2 using the simple relationship

$$
p=g_{s} A_{\mathrm{F}} / A_{\mathrm{BZ}},
$$

where $A_{\mathrm{BZ}}=7.56 \AA^{-2}$ is the area of the first Brillouin zone of graphene and coefficient $g_{s}=2$ due to the spin degeneracy. It is worth noting that a part of the photoemission intensity at the Fermi surface is suppressed by the 'dark corridor' effect due to the influence of the pseudospin $[35,36]$. Fermi surfaces were approximated by circles. The total charge carrier concentration calculated from the Dirac point position from the fit was consistent with that extracted from the Fermi surface area as shown in table 2. Note that the charge density hardly changes with the thickness of the graphene stack, in agreement with the behavior observed with $\mathrm{EG}$ on $\mathrm{SiC}(0001)$ [10]. From Figure 2 the reason for this becomes immediately obvious: even for multilayer QFG (shown here only QFBLG) only the uppermost of the $\pi$-bands is emptied of electrons, i.e. involved in the doping process by equilibrating with the (pyroelectric!) substrate. The additional $\pi$-bands are far enough below the Fermi level to be completely occupied. This clarifies how the position of $E_{\mathrm{D}}$ changes with the addition of graphene layers on $\mathrm{SiC}$. It becomes lower in order to adjust to the hole concentration values of $8.6 \times 10^{12} \mathrm{~cm}^{-2}$ and $6.2 \times 10^{12} \mathrm{~cm}^{-2}$ for QFG on semi-insulating $4 \mathrm{H}-\mathrm{SiC}(0001)$ and $6 \mathrm{H}-\mathrm{SiC}(0001)$, respectively.

The doping level of QFG does, however, depend on the polytype of the substrate. For QFMLG layers on semi-insulating $4 \mathrm{H}-\mathrm{SiC}(0001)$ the hole concentration extracted from the Fermi surface measurement is $8.6 \times 10^{12} \mathrm{~cm}^{-2}$, in comparison to $6.2 \times 10^{12} \mathrm{~cm}^{-2}$ for QFMLG layers on semi-insulating $6 \mathrm{H}-\mathrm{SiC}(0001)$ with the same bulk doping level. This is in very good agreement with the spontaneous polarization doping model [23] which predicts that the doping should be 1.5 times larger for QFMLG on $4 \mathrm{H}-\mathrm{SiC}(0001)$ than for QFMLG on $6 \mathrm{H}-\mathrm{SiC}(0001)$ due to the larger polarization of the substrate [27-30]. This observation strongly corroborates the polarization doping model. We note in passing that this model was also corroborated by a previous report of mild ntype doping of $n=1.2 \times 10^{12} \mathrm{~cm}^{-2}$ for QFMLG on $3 \mathrm{C}-\mathrm{SiC}(001)$ [37], which is confirmed by our measurement in Fig. 2(i), where we get a n-doping of $n=7.4 \times 10^{11} \mathrm{~cm}^{-2}$. This mild n-type doping on cubic substrates is explained by substrate bulk doping below.

Finally we would like to address the influence of the substrate bulk doping level 

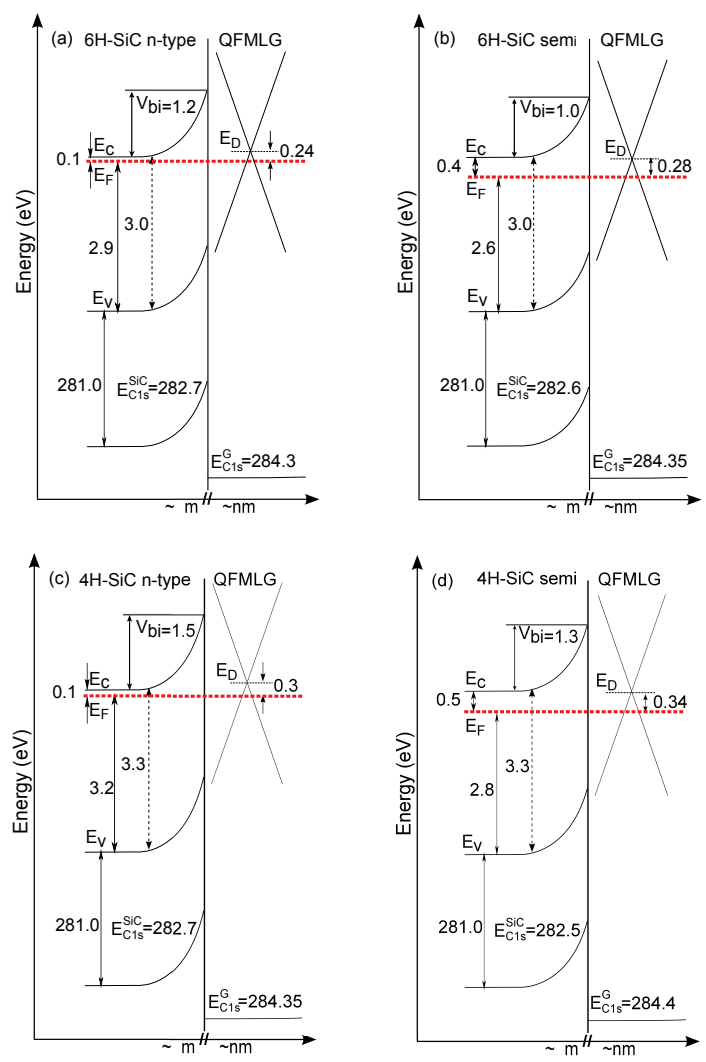

Figure 3. (Color online) Band diagrams of QFMLG on $\mathrm{SiC}(0001)$. (a) n-type 6H$\mathrm{SiC}$, (b) semi-insulating $6 \mathrm{H}-\mathrm{SiC}$, (c) n-type $4 \mathrm{H}-\mathrm{SiC}$, and (d) semi-insulating $6 \mathrm{H}-$ $\mathrm{SiC}$. The bulk doping concentration of the n-type and semi-insulating substrates are $N_{\mathrm{D}} \approx 5.0 \times 10^{17} \mathrm{~cm}^{-3}$ and $N_{\mathrm{D}} \approx 1.0 \times 10^{14} \mathrm{~cm}^{-3}$, respectively. Note that the accuracy of the core level binding energies (approximately $\pm 0.1 \mathrm{eV}$ ) is worse than that of the ARPES measurements (about $\pm 0.02 \mathrm{eV}$ )

on the hole concentration in QFG layers on top. For QFG on both $6 \mathrm{H}-\mathrm{SiC}$ and $4 \mathrm{H}-\mathrm{SiC}$ polytypes, doping was observed to be systematically lower for n-type than for semiinsulating substrates. This effect can be explained by a depletion layer aereal charge density in the $\mathrm{SiC}$. In the spirit of the polarization doping model [23], the negative pseudo polarization charge - which is a fixed number characteristic for the polytype of the $\mathrm{SiC}$ substrate - is balanced by the holes in the QFG layers and a positive space charge in the substrate depletion layer. For the latter one, the aereal charge density $q_{\mathrm{sc}}$ is given by

$$
q_{\mathrm{sc}}=\sqrt{2 \epsilon_{0} \epsilon_{\mathrm{s}} N_{\mathrm{D}} V_{\mathrm{bi}}},
$$

where $\epsilon_{0}=8.84 \times 10^{-14} \mathrm{~F} / \mathrm{cm}$ is the vacuum permeability, $\epsilon_{\mathrm{s}}=10$ the dielectric constant of $\mathrm{SiC}, N_{\mathrm{D}}$ the $\mathrm{SiC}$ doping concentration, and $V_{\mathrm{bi}}$ the surface band bending of the substrate. The latter can be extracted $[38,39]$ from the XPS core level measurements shown in figure 1 . The band alignment between the substrate and graphene for QFMLG on $4 \mathrm{H}$ - and $6 \mathrm{H}-\mathrm{SiC}$ substrates with different bulk donor doping is shown in figure 3. It is worth noting that band bending is almost invariant with 
addition of extra layers, thus calculations below are true for multilayer systems as well.

By substituting the parameters into equation 2 one obtains $q_{\mathrm{sc}}=2.5 \times 10^{12} \mathrm{~cm}^{-2}$ for QFG on n-type $6 \mathrm{H}-\mathrm{SiC}(0001)$ with $N_{\mathrm{D}}=5.0 \times 10^{17} \mathrm{~cm}^{-3}$ and $q_{\mathrm{sc}}=2.0 \times 10^{10} \mathrm{~cm}^{-2}$ for semi-insulating $6 \mathrm{H}-\mathrm{SiC}$ with $N_{\mathrm{D}}=1.0 \times 10^{14} \mathrm{~cm}^{-3}$. Using the same formula we obtain $q_{\mathrm{sc}}=2.8 \times 10^{12} \mathrm{~cm}^{-2}$ for QFG on n-type $4 \mathrm{H}-\mathrm{SiC}(0001)$ with $N_{\mathrm{D}}=$ $5.0 \times 10^{17} \mathrm{~cm}^{-3}$ and $q_{\mathrm{sc}}=2.0 \times 10^{10} \mathrm{~cm}^{-2}$ for semi-insulating $4 \mathrm{H}-\mathrm{SiC}$ with $N_{\mathrm{D}}=$ $1.0 \times 10^{14} \mathrm{~cm}^{-3}$. One should note that the number for bulk carrier concentration contains a rather large uncertainty $\left(1.0 \times 10^{17} \mathrm{~cm}^{-3} \leq N_{\mathrm{D}} \leq 1.0 \times 10^{18} \mathrm{~cm}^{-3}\right.$ for n-type samples). Whereas $q_{\mathrm{sc}}$ is negligible for the semi-insulating substrates, its magnitude for the n-type case is within the uncertainty we have conceded consistent with the difference of around $2.0 \times 10^{12} \mathrm{~cm}^{-2}$ in the aereal hole density of semi-insulating and n-type $\mathrm{SiC}$ substrates (see last column in Table 1). The electron transfer due to the depletion of the n-type $3 \mathrm{C}$-SiC substrate also naturally explains the n-type doping of the QFMLG layer on that substrate.

\section{Conclusions}

Multilayer epitaxial graphene (EG) and quasi-freestanding graphene (QFG) on the Siface of three different polytypes of $\mathrm{SiC}$ was investigated by XPS and ARPES. The hole concentration was observed to depend in a characteristic manner on the $\mathrm{SiC}$ polytype as predicted by the polarization doping model [23]. $4 \mathrm{H}-\mathrm{SiC}$, which has the largest spontaneous polarization induces the largest carrier concentration in QFG, followed by $6 \mathrm{H}-\mathrm{SiC}$ whose polarization and induced hole concentration are approximately 1.5 times smaller. 3C-SiC, which has no spontaneous polarization, induces a negligible n-type doping in QFG which is presumably due to bulk doping, i.e. the depletion of the n-type $\mathrm{SiC}$ substrate. The experiments provide strong evidence in favor of the polarization doping model [23]. The model also provides a roadmap for the choice of substrates for QFG. Clearly, 3C-SiC would be the substrate material of choice because it allows the lowest charge carrier concentration. On the other hand it was shown previously that the deposition parameters for the growth of dielectrics can be tuned to vary surface transfer doping of graphene [40]. This together with the polytype-dependent polarization doping could provide a basis for tuning the charge carrier concentration in QFG on hexagonal $\mathrm{SiC}$ polytypes.

The effect of polarization doping as well as bulk doping on graphene overlayer naturally takes place not only for $\mathrm{QFG}$, but also for $\mathrm{EG}$ on $\mathrm{SiC}$. In that case, the magnitude of this effect is apparently overcompensated by charge transfer from interface states such as, e.g., dangling bonds.

Finally it is worth noting that polarization doping should also be considered for graphene and other 2D materials on pyroelectric substrates. In conjunction with piezoelectric substrates, where the polarization is changed by external forces, this effect could lead to novel applications in electronic devices.

\section{Acknowledgements}

The authors are grateful to Karsten Horn, Hendrik Vita and Stefan Böttcher from the Fritz-Haber-Institute for their ongoing support during beam times at BESSY II as well as for fruitful discussions. The research leading to these results has received funding from the European Union Seventh Framework Programme under grant agreement 
n 604391 Graphene Flagship, from the DFG within the Collaborative Research Centre SFB 953 Synthetic Carbon Allotropes and within the Priority Programme SPP 1459 Graphene (SE 1087/10-1), as well as from the European Science Foundation under the EUROCORES Programme EuroGraphene (SE 1087/9-1).

\section{References}

[1] C. Berger, Z.M. Song, X.B. Li, X.S. Wu, N. Brown, C. Naud, D. Mayo, T.B. Li, J. Hass, A.N. Marchenkov, E.H. Conrad, P.N. First, W.A. de Heer, Science 312, 1191 (2006)

[2] K.V. Emtsev, A. Bostwick, K. Horn, J. Jobst, G.L. Kellogg, L. Ley, J.L. McChesney, T. Ohta, S.A. Reshanov, J. Roehrl, E. Rotenberg, A.K. Schmid, D. Waldmann, H.B. Weber, T. Seyller, Nat. Mater. 8, 203 (2009)

[3] P.N. First, W.A. de Heer, T. Seyller, C. Berger, J.A. Stroscio, J.S. Moon, MRS Bulletin 35, 296 (2010)

[4] J. Moon, D. Curtis, M. Hu, D. Wong, C. McGuire, P. Campbell, G. Jernigan, J. Tedesco, B. VanMil, R. Myers-Ward, C. Eddy, D. Gaskill, IEEE Electron Device Lett. 30, 650 (2009)

[5] Y.-M. Lin, C. Dimitrakopoulos, K. A. Jenkins, D. B. Farmer, H.-Y. Chiu, A. Grill, and P. Avouris, Science 327, 662 (2010)

[6] Y. Wu, K. A. Jenkins, A. Valdes-Garcia, D. B. Farmer, Y. Zhu, A. A. Bol, C. Dimitrakopoulos, W. Zhu, F. Xia, P. Avouris, Y.-M. Lin, Nano Lett. 12, 3062 (2012)

[7] Y. M. Lin, A. Valdes-Garcia, S. J. Han, D. B. Farmer, I. Meric, Y. N. Sun, Y. Q. Wu, C. Dimitrakopoulos, A. Grill, P. Avouris, K. A. Jenkins, Science 332, 1294 (2011)

[8] A. Tzalenchuk, S. Lara-Avila, A. Kalaboukhov, S. Paolillo, M. Syvajarvi, R. Yakimova, O. Kazakova, T. Janssen, V. Falko, S. Kubatkin, Nat. Nanotechnol. 5, 186 (2010)

[9] K. V. Emtsev, F. Speck, Th. Seyller, L. Ley, J. D. Riley. Phys. Rev. B 77, 155303 (2008)

[10] T. Ohta, A. Bostwick, J. McChesney, T. Seyller, K. Horn, E. Rotenberg, Phys. Rev. Lett. 98, $206802(2007)$

[11] A. Mattausch, O. Pankratov, Phys. Rev. Lett. 99, 076802 (2007)

[12] F. Varchon, R. Feng, J. Hass, X. Li, B.N. Nguyen, C. Naud, P. Mallet, J.Y. Veuillen, C. Berger, E. Conrad, L. Magaud, Phys. Rev. Lett 99, 126805 (2007)

[13] S. Goler, C. Coletti, V. Piazza, P. Pingue, F. Colangelo, V. Pellegrini, K. V. Emtsev, S. Forti, U. Starke, F. Beltram, S. Heun, Carbon 51, 249-254 (2103)

[14] S. Kopylov, A. Tzalenchuk, S. Kubatkin, and V. I. Falko, Appl. Phys. lett 97, 112109 (2010)

[15] C. Riedl, C. Coletti, T. Iwasaki, A. A. Zakharov, U. Starke, Phys. Rev. Lett. 103, 246804 (2010)

[16] C. Riedl, C. Coletti, T. Iwasaki, U. Starke, Mater. Sci. Forum 645-648, 623 (2010)

[17] F. Speck, M. Ostler, J. Röhrl, J. Jobst, D. Waldmann, M. Hundhausen, H. Weber, T. Seyller, Mater. Sci. Forum 645-648, 629 (2010)

[18] F. Speck, J. Jobst, F. Fromm, M. Ostler, D. Waldmann, M. Hundhausen, H. B. Weber, Th. Seyller, Appl. Phys. Lett. 99, 12216 (2011)

[19] C. Virojanadara, R. Yakimova, A. A. Zakharov, L. I. Johansson, J. Phys. D: Appl. Phys. 43 374010 (2010)

[20] C. Virojanadara, A. A. Zakharov, R. Yakimova, L. I. Johansson, Surf. Sci. 604, L4 (2010)

[21] H. Tsuchida, I. Kamata, and K. Izumi, Jpn. J. Appl. Phys. Part 2 36, L699 (1997)

[22] N. Sieber, B.F. Mantel, T. Seyller, J. Ristein, L.Ley, T. Heller, D.R. Batchelor, D. Schmeißer, Appl. Phys. Lett. 78, 1216 (2001)

[23] J. Ristein, S. Mammadov, and Th. Seyller. Phys. Rev. Lett. 108, 246104 (2012)

[24] S. B. Austerman, D. A. Berlincour, and H. H. A. Krueger, J. Appl. Phys. 34, 339 (1963)

[25] E. Loh, Phys. Rev. 166, 673 (1968)

[26] Elastic, Piezoelectric and Related Constants of Crystals, O. Madelung (ed.), Landolt-Börnstein Series, Vol III (Springer, Berlin, 1979)

[27] M. Posternak, A. Baldereschi, A. Catellani, and R. Resta, Phys. Rev. Lett. 64, 1777 (1990)

[28] R. Resta, M. Posternak, A. Baldereschi, and A. Catellani, Ferroelectrics 111, 15 (1990)

[29] A. Qteish, V. Heine, and R. J. Needs, Phys. Rev. B 45, 6376 (1992).

[30] J. Soltys, J. Piechota, M. Lopuszynski and S. Krukowski, New J. Phys. 12, 043024 (2010)

[31] M. Ostler, F. Speck, M. Gick, Th. Seyller, Phys. Stat. Sol. B 247, 2924 (2010)

$[32]$ T. Ohta, A. Bostwick, T. Seyller, K. Horn, E. Rotenberg, Science 313, 951 (2006)

[33] E. McCann, V.I. Fal'ko, Phys. Rev. Lett. 96, 086805 (2006)

[34] S. Ulstrup, J. C. Johannsen, F. Cilento, J. A. Miwa, A. Crepaldi, M. Zacchigna, C. Cacho, R. Chapman, E. Springate, S. Mammadov, F. Fromm, C. Raidel, Th. Seyller, F. Parmigiani, M. Grioni, P. D. C. King, Ph. Hofmann, Phys. Rev. Lett. 112, 257401 (2014) 
[35] I. Gierz, J. Henk, H. Höchst, C. R. Ast, K. Kern, Phys. Rev. B 83, 121408 (2011)

[36] Y. Liu, G. Bian, T. Miller, and T.-C. Chiang, Phys. Rev. Lett.107, 166803 (2011)

[37] C. Coletti, K. V. Emtsev, A. A. Zakharov, T. Ouisse, D. Chaussende, U. Starke, Appl. Phys. Lett. 99, 081904 (2011)

[38] Th. Seyller, J. Phys.: Condens. Matter 16, S1755 (2004)

[39] Th. Seyller, K. V. Emtsev, F. Speck, K.-Y. Gao, L. Ley, Mater. Sci. Forum 556-557, 701 (2007)

[40] P. Wehrfritz, F. Fromm, S. Malzer, Th. Seyller, J. Phys. D: Appl. Phys. 47, 305103 (2014) 